\title{
Riesgo psicosocial intralaboral y síndrome de desgaste profesional en psiquiatras de una clínica de Antioquia (Colombia) en el 2020: serie de casos
}

\author{
María Angélica López Rincón ${ }^{1}$ (D) , Julián David Palacio Jaramillo² (D) , Yadilfa Palacios Palacios ${ }^{3}$ (D), \\ Yaline Sánchez Neira ${ }^{4}$
}

\section{RESUMEN}

Introducción: El síndrome de desgaste profesional es un conjunto de signos y síntomas ocasionado por trabajo, fatiga emocional, despersonalización y disminución del sentido de logros personales: por lo cual en este artículo se describen las características demográficas, los riesgos psicosociales intralaborales, el síndrome y el impacto percibido sobre el trabajo por el COVID-19 en un grupo de psiquiatras.

Métodos: Estudio descriptivo de serie de casos en trece psiquiatras incluidos por muestreo no probabilístico a conveniencia que completaron la escala de riesgo psicosocial intralaboral, la escala Maslach Burnout Inventory y una encuesta relacionada con coronavirus SARS-COV-2.

Resultados: Los casos presentan una edad promedio de 35 años con predominio del sexo femenino. Para el $65,1 \%$ de las personas encuestadas, la emoción prevalente fue angustia, temor al contagio e interferencia en el trabajo ante la situación de pandemia. El 69,2\% de los casos percibió que incrementó las actividades intralaborales, con aumento de consultas virtuales, resultados mayores a los obtenidos en estudios, donde los valores van desde el $30 \%$ al 34,5\% en la misma dimensión.

Conclusiones: La prevalencia de síndrome de desgaste profesional fue del 7,7\% en psiquiatras de una clínica de tercer nivel en Antioquia. En los trece casos reportados se encontró un riesgo muy alto, asociado con las condiciones laborales, así como con las actividades laborales ante la actual situación de pandemia por SARS-COV-2.

Palabras clave: desgaste profesional; burnout; psiquiatría; impacto psicosocial; estrés psicológico; trastorno depresivo 1 IPS Salud del Caribe-Sura, Armenia, Quindío, Colombia.

2 Clínica San Juan de Dios, La Ceja, Antioquia, Colombia.

${ }^{3}$ Unidad Santa María IP.S, Quibdó, Chocó, Colombia.

${ }^{4}$ Universidad de Boyacá, Tunja, Boyacá, Colombia.

Autora de Correspondencia: Yaline Sánchez Neira. Correo electrónico: ysanchez@uniboyaca.edu.co

\section{Citar este artículo así:}

López Rincón MA, Palacio Jaramillo JD, Palacios Palacios Y, Sánchez Neira Y. Riesgo psicosocial intralaboral y síndrome de desgaste profesional en psiquiatras de una clínica de Antioquia (Colombia) en el 2020: serie de casos. Rev Investig Salud Univ Boyacá. 2021;8(1):48-62. 


\title{
Intra-Labor Psychosocial Risk and Burnout Syndrome in Psychiatrists at a Clinic in Antioquia (Colombia) in 2020: Case Series
}

\begin{abstract}
Introduction: Burnout syndrome is a set of signs and symptoms related to wear and tear caused by work, emotional fatigue, depersonalization and decreased sense of personal achievement, for which demographic characteristics, intra-occupational psychosocial risks, burnout syndrome and the perceived impact on work by COVID-19 in a group of psychiatrists.

Method: Descriptive study of a series of cases in 13 psychiatrists included by non-probabilistic convenience sampling, who completed the intra-occupational psychosocial risk scale, the Maslach Burnout Inventory scale and a survey related to the SARS-COV-2 coronavirus.

Results: The cases have an average age of 35 years with a predominance of the female sex, $65.1 \%$ of the cases reported that the prevailing emotion was anxiety, fear of contagion and interference at work in the face of a pandemic situation. $69.2 \%$ of the cases, they perceived that intra-labor activities increased, with an increase in virtual consultations, results greater than those obtained in studies, where the values range from $30 \%$ to $34.5 \%$ in the same dimension.

Conclusions: The prevalence of burnout syndrome was $7.7 \%$ in psychiatrists of a third-level clinic in Antioquia, in the thirteen reported cases a very high risk was found associated with working conditions, as well as work activities in the current pandemic situation by SARS-COV-2.
\end{abstract}

Keywords: burnout; psychiatry; psychosocial impact; stress psychological; depressive disorder. 


\title{
Risco psicossocial laboral e síndrome de Burnout em psiquiatras de uma clínica de Antioquia (Colômbia) em 2020: série de casos
}

\begin{abstract}
RESUMO
Introdução: A síndrome de Burnout é um conjunto de sinais e sintomas ocasionado pelo excesso de trabalho, fadiga emocional, despersonalização e diminuição do sentido de logros pessoais: portanto, este artigo descreve as caraterísticas demográficas, os riscos psicossociais no trabalho, a síndrome e o impacto pela COVID-19 percebido no trabalho de um grupo de psiquiatras.

Métodos: Estudo descritivo de série de casos em treze psiquiatras incluídos por amostragem não probabilística a conveniência que completaram a escala de risco psicossocial no trabalho, a Escala Maslach Burnout Inventory é uma pesquisa relacionada com o coronavírus SARS-COV-2.

Resultados: A idade média dos casos é de 35 anos com uma predominância feminina. Para o 65,1 \% das pessoas pesquisadas, a emoção predominante foi à angústia, o medo de contágio e a interferência com o trabalho na situação pandêmica. O 69,2 \% dos casos percebeu o incremento das atividades laborais, com um aumento das consultas virtuais, resultados superiores aos obtidos em estudos, onde os valores variam entre $30 \%$ e $34,5 \%$ na mesma dimensão.
\end{abstract}

Conclusões: A prevalência da síndrome de Burnout foi de 7,7 \% em psiquiatras de uma clinica de terceiro nível em Antioquia. Nos treze casos relatados, foi identificado um risco muito elevado, associado ás condições de trabalho bem como as atividades de trabalho na atual situação pandêmica da SARS-COV-2.

Palavras-chave: burnout; impacto psicossocial; stress psicológico; desordem depressiva. 


\section{INTRODUCCIÓN}

El síndrome de desgaste profesional (burnout) lo definió Herbert Freudenberger en 1974, como un grupo de signos y síntomas físicos, producto de la exposición del individuo al estrés prolongado y directamente relacionado con el ejercicio de su actividad laboral, que aparece luego de un año de trabajo (1), que ocasiona, además, la emergencia de mecanismos de respuesta maladaptativos con el impacto funcional que esto conlleva (2).

En la literatura se han documentado prevalencias del síndrome de desgaste profesional entre un $30 \%$ y un $50 \%$ en médicos, al usar el instrumento Maslach Burnout Inventory (MBI). Se encontró que es más frecuente en psiquiatras que desempeñan funciones en el área asistencial, comparado con personal que tiene a cargo procesos de docencia, administrativas o de investigación (3).

Actualmente, se establece un concepto tridimensional sobre este síndrome, compuesto por la fatiga emocional (desgaste, pérdida de energía y debilitamiento), despersonalización o cinismo (actitudes negativas o inadecuadas hacia los pacientes, con irritabilidad, pérdida de idealismo y retraimiento social) y disminución del sentido de logros personales (4). Para cuantificar el riesgo o diagnosticar el síndrome de desgaste profesional existen múltiples escalas, entre ellas Staff Burnout Scale for Health Professionals (SBS-
HP), Burnout Measure (BM), Shirom-Melamed Burnout Questionnaire y MBI (5).

Existen intervenciones que se asocian con el riesgo psicosocial, que afectan la salud y las funciones del trabajador (6), por lo que en Colombia se debe evaluar el riesgo psicosocial anualmente, y dentro de los métodos sugeridos se encuentra la batería de riesgo psicosocial (7), para definir potenciales intervenciones (8).

Los factores de riesgo psicosocial intralaborales son aquellas distintivos y procesos de una empresa (9), dentro de los cuales se perciben con mayor frecuencia en los trabajadores el prestar servicio público, la monotonía y los procesos repetitivos (10).

En la evaluación de riesgos psicosociales, elaborada por el Ministerio de Protección Social de Colombia en el 2010 y validada por la Universidad Pontificia Javeriana, los autores indican que su formato no debe editarse. Para evaluar los riesgos psicosociales intralaborales incluyen cuatro dominios: las demandas del trabajo, el control sobre el trabajo, la recompensa y el liderazgo y las relaciones sociales, a su vez formadas por un conjunto de dimensiones que actúan como posibles fuentes de riesgo (9). Cuando el trabajador se afecta, ello puede comprometer su salud física y psicológica, y tiene riesgo de sufrir enfermedades mentales como trastornos de adaptación, de ansiedad y depresión (11). 
Los factores estresantes de las actividades diarias del psiquiatra, que requieren interacciones y que en cierta medida son peligrosas, demandan una conexión emocional para que la intervención sea terapéutica (12). A partir de ahí se ha determinado que el $7,6 \%$ de los psiquiatras ha tenido ideación suicida durante su entrenamiento o vida profesional como psiquiatra, comparado con el 2,3\% encontrado en población general con exposición al suicidio. El síndrome de desgaste profesional y el trastorno depresivo fueron los predictores más importantes para la ideación suicida; mientras que el apoyo social, estar casado o vivir acompañado y tener otro médico en la familia fueron factores protectores (13).

Se piensa que el síndrome de desgaste profesional es el resultado de interacciones interpersonales en el lugar de trabajo, factores internos como la personalidad, estrategias de afrontamiento y factores mediadores, como las redes de apoyo (14). Si se presentan síntomas del síndrome, estos deben entenderse como alarmas que deben intervenir para limitar traumas posteriores $o$ ataques emocionales (12).

Con frecuencia, los psiquiatras que requieren atención por su salud mental se automedican, por temor al registro en sus historias clínicas, al estigma o por conocer a los posibles tratantes (15). Los síntomas alteran su juicio clínico y, con ello, la elección que hacen del tratamiento; es ne- cesaria la atención oportuna por el incremento en trastorno depresivo, uso de sustancias psicoactivas y suicidio a las que los profesionales de salud mental no son inmunes (13).

Se han documentado tasas más altas de depresión y agotamiento entre los psiquiatras, comparadas con otras especialidades, debido a que existen muchas presiones culturales relacionadas con las enfermedades mentales y con los propios psiquiatras. Dada la naturaleza de su práctica clínica, tienen mayor riesgo de sufrir depresión, ansiedad y fatiga crónica $(16,17)$. Por lo anterior, el objetivo de este estudio fue evaluar la prevalencia de síndrome de desgaste profesional y los factores de riesgo psicosocial intralaboral entre psiquiatras en una institución de tercer nivel del departamento de Antioquia (Colombia).

\section{SERIE DE CASOS}

Este es un estudio descriptivo que presenta una serie de casos en psiquiatras vinculados laboralmente a una clínica de tercer nivel del departamento de Antioquia (Colombia), cuya población estaba constituida por quince psiquiatras. Para ello se realizó en trece de ellos una técnica de muestreo no probabilístico por conveniencia; además, diligenciaron un consentimiento informado, mediante el cual aceptaban su participación en la investigación, y completaron la escala de riesgo psicosocial intralaboral, la $\mathrm{MBI}$, y una en- 
cuesta relacionada con coronavirus SARS-COV-2. Al final se excluyeron a dos de los psiquiatras, que no firmaron el consentimiento informado.

Posteriormente, se aplicó, mediante encuesta, la Batería de Riesgo Psicosocial Intralaboral, forma A, dirigida a profesionales, compuesta por 123 preguntas con cinco opciones de respuesta, que indica la frecuencia con que suceden los eventos y que mejor refleja el trabajo (siempre, casi siempre, algunas veces, casi nunca, nunca). Dentro de las variables sociodemográficas se tuvieron en cuenta: sexo, edad, estado civil, número de hijos, años de experiencia como psiquiatra, antigüedad en la empresa, horas de la jornada laboral, número de pacientes atendidos por día, tipo de contrato y número de empleos.

Para medir el síndrome de desgaste profesional, se usó la escala MBI, validada en Colombia (18), compuesta por 22 ítems con respuesta tipo Likert de 1 a 7 , que suman a una dimensión específica, y clasifica la presencia del síndrome, cuando es mayor a 9, y 27 en dimensiones de fatiga emocional y despersonalización, respectivamente, y menor a 33, en la dimensión de realización personal.

Debido a la contingencia global por la pandemia ocasionada por el coronavirus SARS-CoV-2 (que produce la enfermedad COVID-19), la salud mental, el efecto en la población en general y los pacientes atendidos por los psiquiatras, se esta- blecieron preguntas que nos permitieron evaluar su impacto en el ejercicio profesional: iconsidera que debido a la contingencia por COVID-19 ha aumentado su trabajo? ¿Cuál es la emoción que ha prevalecido durante esta contingencia? Durante su trabajo, ¿teme constantemente poder contagiarse por el SARS-CoV-2? ¿Interfiere esto en su trabajo? ¿Cambió su forma de trabajo ante la contingencia?

El análisis cuantitativo se llevó a cabo mediante distribuciones de frecuencias para las variables cualitativas, con un nivel de confianza del $95 \%$; mientras que para las variables cuantitativas se utilizaron medidas de tendencia central, como la desviación estándar. Los riesgos psicosociales intralaborales, las variables sociodemográficas, el síndrome de desgaste profesional y el impacto por la pandemia se describieron en tablas con información detallada para cada caso, que se relacionaron por medio de las razones de prevalencia con un intervalo de confianza del 95\%. Para el análisis de datos se utilizó el software Epi Info ${ }^{\mathrm{Tm}}$, versión 7.2.4.0.

La investigación y la recolección de datos comenzaron previo aval del Comité de Bioética de la institución de tercer nivel de salud del departamento de Antioquia. Además, se conservó la confidencialidad, la privacidad y la protección de la identidad de los datos empleados. 


\section{PRESENTACIÓN DE LOS CASOS}

Dentro de la descripción de los signos y la presencia de síntomas como agotamiento físico, mental y emocional, que son característicos para el síndrome de desgaste profesional en personas con tiempos prolongados frente a un estrés ocasionado por actividades laborales, se muestra para el presente estudio que la mayoría de los casos son mujeres (53,8\%). La edad promedio en los participantes fue de 35 años (con una desviación estándar de 6,5). Predominan los solteros (46,2\%), sin hijos (84,60\%). El 53,8\% tiene menos de dos años laborando como especialistas. El $61,5 \%$ solo trabaja en una institución. El 69,2\% tenía un horario menor a 8 horas al día y atiende por día más de 16 pacientes. Por último, el 76,9\% lleva trabajando menos de un año en la institución, y el 92,3\% tiene un contrato temporal, como se observa en la tabla 1.

Tabla 1. Características sociodemográficas de los casos

\begin{tabular}{|c|c|c|c|c|c|c|c|c|c|c|c|c|c|c|}
\hline Caso & 1 & 2 & 3 & 4 & 5 & 6 & 7 & 8 & 9 & 10 & 11 & 12 & 13 & Total n (\%) \\
\hline Edad (años) & 31 & 34 & 29 & 55 & 31 & 35 & 36 & 34 & 33 & 30 & 34 & 38 & 35 & $\begin{array}{l}\leq 35 \text { años: } 10(76,9) \\
\geq 36 \text { años: } 3(23,1)\end{array}$ \\
\hline Sexo & M & $\mathrm{F}$ & $\mathrm{M}$ & $\mathrm{M}$ & M & $\mathrm{F}$ & $\mathrm{F}$ & $\mathrm{F}$ & $\mathrm{F}$ & $\mathrm{F}$ & $\mathrm{M}$ & $\mathrm{M}$ & $\mathrm{F}$ & $\begin{array}{l}\text { F: } 7(53,8) \\
\text { M: } 6(46,2)\end{array}$ \\
\hline Estado civil & S & $\mathrm{C}$ & S & $\mathrm{D}$ & S & $\mathrm{S}$ & S & $\mathrm{C}$ & $\mathrm{U}$ & $\mathrm{U}$ & $\mathrm{D}$ & S & $\mathrm{U}$ & $\begin{array}{c}\text { S: } 6(46,2) \\
\text { UL: } 3(23,0) \\
\text { C: } 2(15,4) \\
\text { D: } 2(15,4)\end{array}$ \\
\hline Hijos & 0 & 0 & 0 & 0 & 0 & 0 & 0 & 1 & 0 & 0 & 0 & 0 & 1 & $\begin{array}{c}0: 11(84,6) \\
1: 2(15,4)\end{array}$ \\
\hline Años de graduado & 1 & 3 & 1 & 21 & 2 & 4 & 6 & 4 & 2 & 1 & 2 & 4 & 2 & $\begin{array}{l}\leq 2 \text { años: } 7(53,8) \\
\geq 3 \text { años: } 6(46,2)\end{array}$ \\
\hline Años en la institución & 1 & 3 & 1 & 1 & 1 & 1 & 1 & 3 & 2 & 1 & 1 & 0 & 1 & $\begin{array}{l}\leq 1 \text { año: } 10(76,9) \\
\geq 2 \text { años: } 3(23,1)\end{array}$ \\
\hline Horas por semana de trabajo & 24 & 48 & 48 & 48 & 48 & 48 & 10 & 27 & 48 & 48 & 48 & 48 & 24 & $\begin{array}{l}48 \text { horas: } 9(69,2) \\
<48 \text { horas: } 4(30,8)\end{array}$ \\
\hline Número de pacientes por jornada & 10 & 17 & 16 & 15 & 18 & 18 & 15 & 16 & 20 & 18 & 18 & 16 & 12 & $\begin{array}{l}\leq 15: 4(30,8) \\
\geq 16: 9(69,2)\end{array}$ \\
\hline Tipo de contrato & $\mathrm{T}$ & $\mathrm{T}$ & $\mathrm{T}$ & $\mathrm{T}$ & $\mathrm{T}$ & $\mathrm{T}$ & $\mathrm{T}$ & $\mathrm{T}$ & $\mathrm{T}$ & $\mathrm{T}$ & $\mathrm{T}$ & $\mathrm{T}$ & I & $\begin{array}{c}\text { I: } 1(7,7) \\
\text { T: } 12(92,3)\end{array}$ \\
\hline Número de sitios de trabajo & 2 & 1 & 1 & 1 & 1 & 2 & 2 & 2 & 1 & 2 & 1 & 1 & 1 & $\begin{array}{l}1: 8(61,5) \\
2: 5(38,5)\end{array}$ \\
\hline
\end{tabular}

M: masculino, F: femenino, S: soltero, D: divorciado, T: temporal, I: indefinido. 
En la clasificación, en una situación intralaboral se lograron evaluar los dominios de demandas del trabajo, control sobre el trabajo y recompensa para todos los participantes. En cuanto al dominio de liderazgo y relaciones sociales, el $30,8 \%$ de los psiquiatras se considera jefes de otras personas en su trabajo, por lo que en la encuesta esta dimensión, denominada relación con los colaboradores, no fue calificada por el $69,2 \%$ de los participantes, situación que explica la falta de calificación del riesgo, según el puntaje total para todos los participantes.

En el caso 11 hay dominio de recompensa $\sin$ riesgo; en el resto se evidencia algún riesgo en los dominios evaluados. En aquellos quienes completaron toda la batería de riesgo psicosocial intralaboral (casos 2, 5, 10 y 12) se encontró un puntaje total de riesgo muy alto (tabla 2); en los demás casos, no fue calificado el dominio y no suma puntaje. Por lo tanto, ello limita el cálculo del puntaje transformado de los factores de riesgo psicosocial intralaboral en este estudio.

De la escala $\mathrm{MBI}$ se encontró que la prevalencia fue del 7,7\%, y fue el caso 5 el que tiene síndrome de desgaste profesional (tabla 3). El 38,5\% tiene dos dimensiones en riesgo alto (casos 4, 6 , 9,10 y 13 ); el $30,8 \%$, una dimensión en riesgo alto (casos 2, 3, 8 y 12); mientras que para los casos 1,7 y 11 no se registraron dimensiones en riesgo alto, y conforman el $23,1 \%$ de la muestra. El riesgo alto se encontró en el $69,2 \%$ de los casos para la dimensión de agotamiento emocional; en

Tabla 2. Factores de riesgo psicosocial intralaboral en los casos

\begin{tabular}{|c|c|c|c|c|c|c|c|c|c|c|c|c|c|}
\hline Caso & 1 & 2 & 3 & 4 & 5 & 6 & 7 & 8 & 9 & 10 & 11 & 12 & 13 \\
\hline DO. Recompensas & 3 & 3 & 4 & 4 & 4 & 1 & 1 & 4 & 4 & 4 & 0 & 4 & 2 \\
\hline DO. Demandas del trabajo & 4 & 2 & 3 & 3 & 4 & 4 & 2 & 2 & 4 & 3 & 1 & 4 & 2 \\
\hline DO. Control sobre el trabajo & 4 & 4 & 4 & 3 & 4 & 1 & 2 & 3 & 4 & 4 & 1 & 4 & 4 \\
\hline DO. Liderazgo y relaciones sociales & - & 4 & - & - & 4 & - & - & - & - & 4 & - & 4 & - \\
\hline DI. Retroalimentación del desempeño & 4 & 4 & 3 & 4 & 4 & 0 & 4 & 2 & 3 & 4 & 1 & 4 & 4 \\
\hline DI. Relaciones sociales en el trabajo & 0 & 3 & 2 & 3 & 3 & 1 & 1 & 2 & 3 & 3 & 3 & 3 & 1 \\
\hline DI. Características del liderazgo & 2 & 4 & 4 & 4 & 4 & 0 & 3 & 4 & 4 & 4 & 1 & 4 & 4 \\
\hline DI. Relación con los colaboradores & - & 3 & - & - & 1 & - & - & - & - & 2 & - & 3 & - \\
\hline Puntaje total & - & 4 & - & - & 4 & - & - & - & - & 4 & - & 4 & - \\
\hline
\end{tabular}

0: Sin riesgo, 1: Riesgo bajo, 2: riesgo medio, 3: riesgo alto, 4: riesgo muy alto.

DO: dominio, DI: dimensión. 
Tabla 3. Escala MBI

\begin{tabular}{lcccccccccccccc}
\hline \multicolumn{1}{c}{ Caso } & $\mathbf{1}$ & $\mathbf{2}$ & $\mathbf{3}$ & $\mathbf{4}$ & $\mathbf{5}$ & $\mathbf{6}$ & $\mathbf{7}$ & $\mathbf{8}$ & $\mathbf{9}$ & $\mathbf{1 0}$ & $\mathbf{1 1}$ & $\mathbf{1 2}$ & $\mathbf{1 3}$ & Total n (\%) \\
\hline Falta de realización & 1 & 1 & 2 & 1 & 3 & 1 & 2 & 3 & 2 & 3 & 1 & 1 & 2 & \\
\hline Despersonalización & 1 & 2 & 2 & 3 & 3 & 3 & 2 & 1 & 3 & 2 & 1 & 2 & 3 & \\
\hline Agotamiento & 1 & 3 & 3 & 3 & 3 & 3 & 1 & 2 & 3 & 3 & 1 & 3 & 3 & $1(7,7)$ \\
\hline Agotamiento profesional & - & - & - & - & $\mathrm{x}$ & - & - & - & - & - & - & - & - & \\
\hline
\end{tabular}

MBI: Maslach Burnout Inventory; x: presenta.

1: bajo, 2: medio, 3: alto.

Tabla 4. Interferencia por coronavirus SARS-CoV-2 en el trabajo

\begin{tabular}{|c|c|c|c|c|c|c|c|c|c|c|c|c|c|c|}
\hline Caso & 1 & 2 & 3 & 4 & 5 & 6 & 7 & 8 & 9 & 10 & 11 & 12 & 13 & Total n (\%) \\
\hline Aumento de trabajo & $\mathrm{x}$ & - & $\mathrm{x}$ & - & $\mathrm{x}$ & $\mathrm{x}$ & $\mathrm{x}$ & - & $\mathrm{x}$ & $\mathrm{x}$ & $\mathrm{x}$ & - & $\mathrm{x}$ & $9(69,2)$ \\
\hline Emoción prevalente & $\mathrm{N}$ & An & An & $\mathrm{T}$ & An & An & An & An & $\mathrm{T}$ & An & $\mathrm{Al}$ & $\mathrm{N}$ & An & $\begin{array}{c}\mathrm{N}: 2(15,4) \\
\text { Al: } 1(7,7) \\
\text { An: } 8(61,5) \\
\text { T: } 2(15,4)\end{array}$ \\
\hline Temor al contagio & - & - & - & $\mathrm{x}$ & $\mathrm{x}$ & $\mathrm{x}$ & $\mathrm{x}$ & $\mathrm{x}$ & $\mathrm{x}$ & $\mathrm{x}$ & - & - & $\mathrm{x}$ & $8(61,5)$ \\
\hline Interferencia por temor al contagio & - & $\mathrm{x}$ & $\mathrm{x}$ & $\mathrm{x}$ & $\mathrm{x}$ & $\mathrm{x}$ & - & - & $\mathrm{x}$ & $\mathrm{x}$ & $\mathrm{x}$ & - & - & $8(61,5)$ \\
\hline Cambio en forma de trabajo & $\mathrm{x}$ & $\mathrm{x}$ & $\mathrm{x}$ & $\mathrm{x}$ & $\mathrm{x}$ & $\mathrm{x}$ & $\mathrm{x}$ & $\mathrm{x}$ & $\mathrm{x}$ & $\mathrm{x}$ & $\mathrm{x}$ & $\mathrm{x}$ & $\mathrm{x}$ & $13(100)$ \\
\hline
\end{tabular}

N: ninguna; An: angustia; T: tristeza; Al: alegría; $x$ : presente.

el 38,5\% para la dimensión de despersonalización, y en el $23 \%$ en la dimensión de falta de realización personal.

Por la pandemia debida al virus SARS-CoV-2, el $69,2 \%$ de los casos notó un aumento en su trabajo; el 65,1 \% de ellos informó que la emoción prevalente fue la angustia, y esta misma proporción reportó temor al contagio e interferencia en el trabajo. Todos los casos refieren que por motivo de la pandemia percibieron cambios en su forma de trabajo; resaltaron principalmente el paso de la atención presencial a la virtualidad o teleasistencia, que implica incremento de actividades que antes no realizaban, con disminución en el tiempo de intervención terapéutica al paciente durante la consulta, por las limitaciones propias del tipo de atención (tabla 4). 


\section{DISCUSIÓN}

En muestras de médicos se encuentra el predominio de mujeres, población soltera y sin hijos, como se evidencia en la serie de casos. El promedio de edad y el tiempo de trabajo son más bajos y las jornadas laborales son más extensas (19-22). Las características sociodemográficas de la población eran similares en cuanto a edad, estado civil, número de hijos y años de experiencia, en relación con un estudio realizado por Del Carpio et al. (23), en el 2019, en población peruana.

En cuanto al síndrome de desgaste profesional, para la serie de casos afectó al $16,6 \%$ de los hombres, comparado con ninguna mujer, situación que difiere con otros reportes en la literatura, donde se evidencia más frecuencia en población femenina $(13,19,20)$. Para la escala MBI, en la dimensión de agotamiento emocional, el riesgo alto fue mayor comparado con el resto de los estudios (12,19-24). En cuanto a la dimensión de despersonalización, el riesgo alto es similar a los estudios realizados por Escribá et al. $(24,25)$, con valores del $49,4 \%$ hasta el $57,8 \%$, respectivamente. Por otro lado, se evidencia que los resultados obtenidos por Rotstein et al. (12), Aranda-Beltrán et al. (19), Castañeda-Aguilera et al. (20), Gan et al. (21) y Bruschini et al. (22) son mayores en cuanto a la misma dimensión. Para la dimensión de falta de realización personal, los datos obtenidos para el riesgo alto fueron similares a los estudios realizados por Escribá et al. (24), Gan et al. (21) y Rotstein et al. (12), valores que van desde el $30 \%$ al $34,5 \%$, comparado con el $23 \%$ obtenido en este estudio, lo cual difiere significativamente de lo encontrado por el resto de los autores $(19,20,22,25)$.

Por consiguiente, en este estudio, la dimensión con menor puntaje para riesgo alto de burnout fue la falta de realización personal. Esto puede ir en relación con lo descrito por Kumar (14), donde no se encuentra relación inversa de satisfacción y estrés laboral, documentado en otras profesiones. Así, se piensa que los psiquiatras siguen disfrutando de su trabajo, a pesar de experimentar el síndrome.

Respecto a la batería de riesgo psicosocial, para identificación de factores intralaborales, llama la atención que, en los casos de estudio, no se consideran jefes de otras personas al interior de la organización, al comparar con un estudio realizado en una institución de tercer nivel salud en Cali, el cual pudo calcular el factor de riesgo intralaboral total. Sin embargo, se encuentra un comportamiento similar en el riesgo muy alto con mayor frecuencia en los dominios de las demandas del trabajo y el control sobre el trabajo (26). Adicionalmente en el presente estudio, se encontró que había compromiso de riesgo muy alto en recompensas y dimensiones de características del liderazgo y la retroalimentación del desempeño. 
Se ha identificado entre psiquiatras que la satisfacción laboral está directamente relacionada con la cooperación, la tasa de realización personal y menor riesgo de agotamiento (27), situación que pone en riesgo a nuestra población, ya que se encontró que el riesgo es alto en la dimensión de las relaciones sociales en el trabajo.

El agotamiento emocional relacionado con el trabajo se asocia con niveles altos de estrés, impacto psicológico por el suicidio de pacientes, servicios disponibles ocupados, recursos limitados, poco tiempo para su atención, pacientes difíciles, desconocimiento de la naturaleza de las enfermedades mentales, desinformación generalizada al respecto y expectativas de la sociedad sobre la función del psiquiatra $(28,29)$. Dicha situación se ha venido agudizando en lo encontrado en la encuesta de COVID-19, realizada en la población de estudio.

La sobrecarga laboral y el mal gerenciamiento entorpecen el entorno laboral y generan riesgos que impactan negativamente el desempeño del trabajador de salud, por lo cual se encontraron dimensiones en alto riesgo que pueden hablar de ello (30). La asociación entre condiciones psicosociales y la violencia que padece el médico de parte de pacientes y acompañantes son inherentes a las funciones del psiquiatra (23).
Una forma de disminuir el riesgo es mejorar y facilitar la comunicación entre empleador y empleado, que permitan compartir las dificultades diarias y evitar la sobrecarga. Las horas de trabajo, más allá que la carga laboral, puede estar asociada con dificultades para los profesionales. Dentro de los factores protectores se han descrito el espíritu de cooperación, que permite un entorno profesional para el logro de objetivos comunes (22), situación que se encuentra comprometida en la mayoría de los casos, dado el riesgo alto en la dimensión de relaciones sociales en el trabajo.

Hay factores de riesgo específicos entre psiquiatras, dentro de ellos la inestabilidad laboral, como se evidencia en el 93,7\% los casos reportados con contrato temporal; las escasas posibilidades de ofrecer psicoterapia; no sentir reconocimiento por el trabajo realizado; las opiniones que existen entre los valores individuales y los del lugar de trabajo; así como tener una red de apoyo laboral poco eficiente, además de los recursos limitados, que interfieren en la posibilidad de realizar intervenciones requeridas (3).

Castañeda-Aguilera et al. (20) han referido factores de riesgo para síndrome de desgaste profesional como ser mujer, tener menos de cuarenta años, una pareja que no trabaja, no tener hijos, tener contratación definida y laborar más de cuatro horas en otro lugar de trabajo y menos de diez años de antigüedad profesional; así como una 
menor satisfacción laboral y exposición a violencia en el lugar de trabajo (21). Lo anterior, a diferencia del meta-análisis de Bruschini et al. (22), donde identifican que no hay relación significativa entre variables sociodemográficas con dimensiones del síndrome.

Es de gran interés correlacionar los diferentes factores de riesgo psicosocial intralaboral, las características sociodemográficas y las dimensiones de síndrome de desgaste profesional, por lo cual se recomienda que se lleven a cabo estudios que permitan incluir una mayor población, para identificar los posibles riesgos del síndrome y definir intervenciones que disminuyan el riesgo de padecerlo.

Dentro de las limitaciones del presente estudio, se resalta que la mayoría de los participantes de la encuesta no calificaron la dimensión denominada relación con los colaboradores-subordinados, y ello no sumó puntaje al dominio y restringió el cálculo del puntaje transformado de los factores de riesgo psicosocial intralaboral en este estudio.

\section{CONCLUSIONES}

Este artículo determinó que la prevalencia de síndrome de desgaste profesional en un grupo de psiquiatras de una clínica de tercer nivel fue del 7,7\%, según la aplicación de la escala MBI. La mayoría de los psiquiatras tenían dos dimensiones que están en riesgo alto. Los casos de estudio no se consideran jefes o que tengan personal a cargo, lo que limitó la evaluación de riesgo psicosocial intralaboral, según el puntaje total usando la batería disponible por el Ministerio de la Protección Social; sin embargo, en los cuatro casos reportados que completaron toda la encuesta se encontró un riesgo muy alto asociado a las condiciones laborales. La pandemia debida al virus SARS-CoV-2 cambió la forma de trabajo de todos los psiquiatras, lo que reportó un aumento en el trabajo y generó algún impacto emocional en la mayoría de los casos.

\section{CONFLICTO DE INTERESES}

No existen intereses institucionales, lucrativos o personales relacionados con el desarrollo del trabajo de investigación. La información tanto de los participantes como de las instituciones se trató confidencialmente, mediante la codificación de la información de cada participante para proteger su privacidad.

\section{FINANCIACIÓN}

La financiación del presente estudio contó con el aporte de las instituciones participantes y de los investigadores de la Universidad de Boyacá.

\section{REFERENCIAS}

1. Freudenberger, Herbert J. Staff burn-out. J Soc Issues. 1974;90(1):159-65. 
2. McFarland DC, Hlubocky F, Riba M. Update on addressing mental health and burnout in physicians: what is the role for psychiatry? Curr Psychiatry Rep. 2019;21(11). http://dx.doi. org/10.1007/s11920-019-1100-6

3. Tejada P, Gómez V. Prevalencia y factores demográficos y laborales asociados al burnout de psiquiatras en Colombia. Univ Psychol. 2012;11(3):863-73. http://dx.doi. org/10.11144/javeriana.upsy11-3.pfdl

4. Olivares Faúndez V. Laudatio: Dra. Christina Maslach, comprendiendo el burnout. Cienc Trab. 2017 Apr;19(58):59-63. https://doi. org/10.4067/S0718-24492017000100059

5. Torres E, González L, Gutiérrez V. Análisis del burnout y sus escalas de medición. Prax Investig Redie [internet]. 2019;11(20):XX. Disponible en: https://dialnet.unirioja.es/servlet/articulo?codigo $=6951593$ teorica/Dialnet-AnalisisDelBurnoutYSusEscalasDeMedicion-6951593.pdf

6. Resolución 2646/de 17 de julio 2008 del Ministerio de Salud y Protección Social de Colombia [internet], por la cual se establecen disposiciones y se definen responsabilidades para la identificación, prevención, intervención y monitoreo permanente de la exposición a factores de riesgo psicosocial.... Disponible en: http://www.saludcapital.gov.co/Documen- tos Salud Ocupacional/RESOL. 2646 DE 2008 RIESGO PSICOSOCIAL.pdf

7. Resolución 2404/de 22 de julio de 2019 del Ministerio de Trabajo [internet], por la cual se adopta la batería de instrumentos para la evaluación de factores de riesgo psicosocial... Disponible en: http://www.mintrabajo. gov.co/documents/20147/59995826/Resolucion $+2404+$ de +2019 - + Adopcion + bateria + riesgo + psicosocial\% $2 \mathrm{C}+$ guia $+\mathrm{y}+$ protocolos.pdf

8. Prince M, Patel V, Saxena S, Maj M, Maselko J, Phillips $M R$, et al. No health without mental health. Lancet. 2007;370(9590):85977. https://doi.org/10.1016/S01406736(07)61238-0

9. Ministerio de la Protección Social de Colombia. Batería de instrumentos para la evaluación de factores de riesgo psicosocial [internet]. Bogotá; 2010. Disponible en: https://www. protegerips.com/images/bateria-riesgo-psicosocial-1.pdf

10. Ministerio del Trabajo de Colombia. Informe ejecutivo-II. Encuesta Nacional de Condiciones de Seguridad y Salud en el Trabajo en el Sistema General de Riesgos Laborales [Internet]. Bogotá; 2013. Disponible en: https://fasecolda.com/cms/wp-content/uploads/2019/08/ 
ii-encuesta-nacional-seguridad-salud-trabajo-2013.pdf

11. Albarrán V, Geldres V, Paredes $P$, Ramirez $O$, Ruiz F, Palomino JC. Validación de la batería de instrumentos para la evaluación de factores de riesgo psicosocial. Horiz Médico. 2018;18(1):59-67. https://doi.org/10.24265/ horizmed.2018.v18n1.09

12. Rotstein $S$, Hudaib AR, Facey $A$, Kulkarni J. Psychiatrist burnout: a meta-analysis of Maslach Burnout Inventory means. Australas Psychiatry. 2019;27(3):249-54. https://doi. org/10.1177/1039856219833800

13. Fresán $A$, Yoldi-Negrete $M$, Robles-García $R$, Tovilla-Zárate CA, Suárez-Mendoza A. Professional adversities and protective factors associated with suicidal ideation in Mexican psychiatrists. Arch Med Res. 2019;50(8):484-9. https://doi.org/10.1016/j.arcmed.2019,11.010

14. Kumar S. Burnout in psychiatrists. World Psychiatry [internet]. 2007;6(3):186-9. Disponible en: https://www.ncbi.nlm.nih.gov/pmc/articles/PMC2175073/

15. Balon R. Psychiatrist attitudes toward self-treatment of their own depression. Psychother Psychosom. 2007;76(5):306-10. https://doi.org/10.1159/000104707
16. Looney JG, Harding RK, Blotcky MJ, Barnhart FD. Psychiatrists' transition from training to career: stress and mastery. Am J Psychiatry. 1980;137(1):32-6. https://doi.org/10.1176/ ajp.137.1.32

17. Prosser D, Johnson S, Kuipers E, Szmukler G, Bebbington P, Thornicroft G. Mental health, "burnout" and job satisfaction among hospital and community-based mental health staff. Br J Psychiatry. 1996;169(3):334-7. https://doi. org/10.1192/bjp.169.3.334

18. Córdoba L, Tamayo JA, González MA, Martínez MI, Rosales A, Barbato SH. Adaptation and validation of the Maslach Burnout Inventory-human services survey in Cali, Colombia. Colomb Med. 2011;42(3):286-93. https://doi. org/10.25100/cm.v42i3.874

19. Aranda-Beltrán C, Pando-Moreno M, Torres-López T, Salazar-Estrada O, Franco-Chávez $\mathrm{S}$. Factores psicosociales y síndrome de burnout en médicos de familia. México. An Fac Med (Perú). 2013;66(3):225. https://doi. org/10.15381/anales.v66i3.1346

20. Castañeda-Aguilera E, García de-Alba-García JE. Síndrome de desgaste profesional (burnout) en médicos cirujanos especialistas: prevalencia y factores de riesgo. Cir Cir. 2020 May 4;88(3):354-60. http://dx.doi. org/10.24875/CIRU.19001502 
21. Gan Y, Jiang $H$, Li L, Yang Y, Wang C, Liu J, et al. Prevalence of burnout and associated factors among general practitioners in Hubei, China: A cross-sectional study. BMC Public Health. 2019;19(1):1-9. https://doi.org/10.1186/ s12889-019-7755-4

22. Bruschini M, Carli A, Burla F. Burnout and work-related stress in Italian rehabilitation professionals: A comparison of physiotherapists, speech therapists and occupational therapists. Work. 2018;59(1):121-9. https://doi. org/10.3233/WOR-172657

23. Del Carpio Toya AM, Arias Gallegos WL, Caycho-Rodríguez T. Burnout syndrome in medicine men from Arequipa city (Peru). Rev Chil Neuropsiquiatr. 2019;57(2):139-48. https:// doi.org/10.4067/S0717-92272019000200139

24. Escribà-Agüir V, Bernabé-Muñoz Y. Exigencias laborales psicológicas percibidas por médicos especialistas hospitalarios. Gac Sanit. 2002;16(6):487-96. https://doi.org/10.1016/ s0213-9111(02)71969-9

25. Escribà-Agüir V, Artazcoz L, Pérez-Hoyos S. Efecto del ambiente psicosocial y de la satisfacción laboral en el síndrome de burnout en médicos especialistas. Gac Sanit. 2002;22(4):300-8. https://doi. org/10.1157/13125351
26. Bobadilla C, Garzón L, Charria V. Identificación de factores de riesgo psicosocial en una clínica de alta complejidad. Psicol desde el Caribe. 2018; 35:1-14. https://doi.org/10.14482/ psdc.35.2.7650

27. Baumgardt J, Moock J, Rössler W, Kawohl W. Aspects of sustainability: cooperation, job satisfaction, and burnout among Swiss psychiatrists. Front Public Heal. 2015;3(feb):1-8. https://doi.org/10.3389/fpubh.2015.00025

28. Aguilera EC, de Alba García JEG. Prevalencia del síndrome de agotamiento profesional (burnout) en médicos familiares mexicanos: análisis de factores de riesgo. Rev Colomb Psiquiatr. 2010;39(1):67-84. https://doi. org/10.1016/s0034-7450(14)60237-7

29. Deahl M, Turner T. General psychiatry in noman's land. Br J Psychiatry. 1997;171:6-8. https://doi.org/10.1192/bjp.171.1.6

30. Santana L de L, Sarquis LMM, Miranda FMDA. Psychosocial risks and the health of health workers: reflections on Brazilian labor reform. Rev Bras Enferm. 2020;73(1 Suppl 1):e20190092. https://doi.org/10.1590/00347167-2019-0092

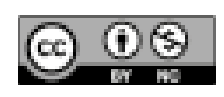

Esta obra está bajo una licencia de Creative Commons Reconocimiento-NoComercial 4.0 Internacional 